\title{
Design of Absorptive Microwave Filters using Allpass Networks in a Parallel-Cascade Configuration
}

\author{
Andrew C. Guyette ${ }^{1}$, Ian C. Hunter ${ }^{2}$, and Roger D. Pollard ${ }^{2}$ \\ ${ }^{1}$ Microwave Technology Branch, Electronics Science and Technology Division, \\ Naval Research Laboratory, Washington, DC, 20375, USA \\ ${ }^{2}$ Institute of Microwaves and Photonics, School of Electronic and Electrical Engineering, \\ University of Leeds, Leeds, LS2 9JT, UK
}

\begin{abstract}
A new approach to designing absorptive microwave filters using allpass networks in a parallel-cascade configuration is presented. A signal is divided into two paths, each comprised of a number of cascaded allpass networks. The two signals are then recombined, and frequency selectivity results from phase cancellation. It is shown that this approach is especially useful for dissipative loss compensation. A general method for designing the microwave allpass networks is given. Bandpass and bandstop microstrip prototypes were designed, built, and tested.
\end{abstract}

Index Terms - Microwave filters, microstrip filters.

\section{INTRODUCTION}

Filters are required in most microwave systems. Typically, microwave filters are reflective in nature - energy is differentially reflected in order to realize frequency selectivity. For some applications, however, a large reflected signal is undesirable, and either attenuators or isolators must be used in order to achieve an acceptable impedance match. An alternative is to use an absorptive filter, where energy is selectively absorbed instead of reflected.

Absorptive filters are typically implemented by cancelling out the reflections of two identical reflective filters using matched power dividers. In the alternative approach proposed in this paper, a similar topology is used, except the transmission responses of two different allpass filters are combined and selectivity arises from phase cancellation (Fig. 1). Advantages of this approach include ease of realizing complex transfer functions, dissipative loss compensation, and relatively straight-forward tuning, as each allpass filter can be decomposed into a cascade of lower-order allpass networks, each independently contributing one or more poles. The only requirement on the transfer function is that it be symmetrical.

\section{BASIC APPROACH}

A symmetrical network can be characterized by its responses to even- and odd-mode excitations, which are equivalent to placing an open or a short circuit along the line of symmetry. The S-parameters of a symmetrical network can be expressed in terms of the even- and odd-mode reflection coefficients $S_{e}$ and $S_{o}[1]$ :

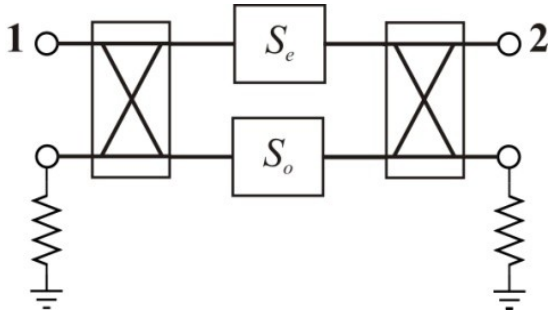

Fig. 1. The proposed absorptive filter architecture. Two allpass filters realize the even- and odd-mode reflection coefficients of a symmetrical transfer function, which is reconstructed by adding or subtracting the allpass responses with matched power dividers.

$$
\begin{gathered}
S_{11}=\frac{S_{e}+S_{o}}{2} \\
S_{12}=\frac{S_{e}-S_{o}}{2}
\end{gathered}
$$

Assume for the moment that the network is lossless. $S_{e}$ and $S_{o}$ are the reflection coefficients of lossless (i.e. reactance) one-port networks, and so the corresponding magnitudes $\left|S_{e}\right|^{2}$ and $\left|S_{o}\right|^{2}$ are equal to unity for all frequencies. $S_{e}$ and $S_{o}$ must therefore be comprised of complementary poles and zeros:

$$
\begin{aligned}
& S_{e}=\frac{\left(p-z_{e 1}^{*}\right)\left(p-z_{e 2}^{*}\right) \cdots}{\left(p+z_{e 1}\right)\left(p+z_{e 2}\right) \cdots} \\
& S_{o}=\frac{\left(p-z_{o 1}^{*}\right)\left(p-z_{o 2}^{*}\right) \cdots}{\left(p+z_{o 1}\right)\left(p+z_{o 2}\right) \cdots}
\end{aligned}
$$

Where $p$ is the complex frequency variable and the constants $z$ are in general complex with positive real parts. It is apparent upon inspection of (3) and (4) that the functions $S_{e}$ and $S_{o}$ may be realized in a transmission-mode fashion as a cascade of allpass networks, each contributing one or more pole/zero pairs. $S_{11}$ and $S_{12}$ are then realized by adding or subtracting the responses of the two networks, as per (1) and (2). This is readily done using matched power dividers (Fig. 1). It should be noted that a complimentary diplexer is created by replacing the resistive load of the output power divider with an additional port, with one output port providing the sum of the two paths $\left(S_{11}\right)$, and the other the difference $\left(S_{12}\right)$. 


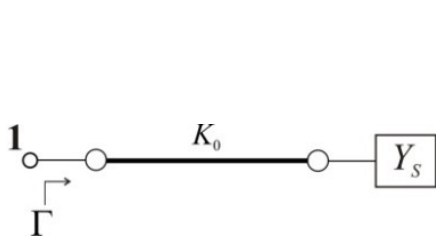

(a)

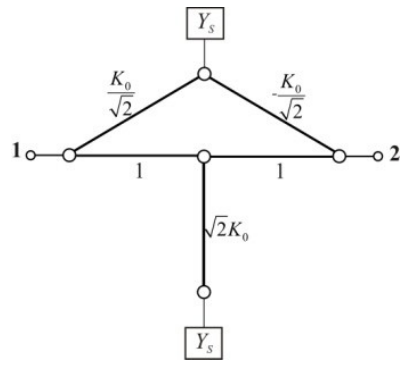

(c)

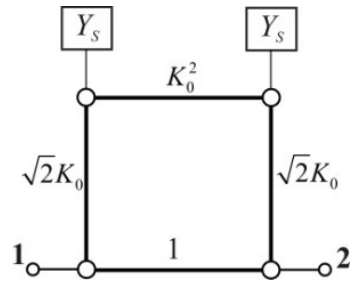

(b)

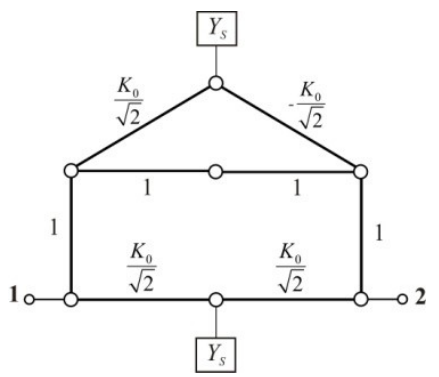

(d)
Fig. 2. (a) Subnetwork, comprised of an admittance inverter $K_{0}$ and an admittance $Y_{S}$. (b) Allpass topology with $\emptyset=\pi / 2$ (hybrid). (c) Allpass topology with $\varnothing=\pi$ (bridged-T). (d) Allpass topology with $\emptyset=2 \pi$.

\section{MicRowaVe AlLPASS NETWORKS}

An ideal lossless allpass network has the property of passing all frequencies with zero attenuation, and thus must present a perfect match at all frequencies $\left(S_{11}=0\right)$. Under this condition (1) and (2) become:

$$
S_{12}=S_{e}=-S_{o}
$$

Eq. (5) implies that allpass networks can be constructed from two identical one-port subnetworks. Assume that these subnetworks are of the form shown in Fig. 2a, where $Y_{S}$ is an admittance, and the input coupling is represented by an admittance inverter of value $K_{0}$. The reflection coefficient $\Gamma$ of this network is:

$$
\Gamma=\frac{Y_{s}-K_{0}^{2}}{Y_{S}+K_{0}^{2}}
$$

Now assume that this subnetwork is part of a network with a transfer response $S_{12}$ equal to $\Gamma$, but with an arbitrary phase shift $\emptyset$ and magnitude $A$ :

$$
S_{12}=A e^{j \varnothing} \Gamma
$$

Consider the case where $\varnothing=\pi / 2$ and $A=1$. The even-mode admittance $Y_{e}$ is the bilinear transform of $S_{e}$. Using (5):

$$
Y_{e}=\frac{1}{Y_{o}}=\frac{1-S_{12}}{1+S_{12}}=\frac{1-j \Gamma}{1+j \Gamma}=\frac{Y_{S}(1-j)+K_{0}^{2}(1+j)}{Y_{S}(1+j)+K_{0}^{2}(1-j)}
$$

Expanding (8) in terms of $Y_{s}$ gives:

$$
Y_{e}=-j+\frac{2 K_{0}^{2}}{Y_{S}-j K_{0}^{2}}, \quad Y_{o}=j+\frac{2 K_{0}^{2}}{Y_{S}+j K_{0}^{2}}
$$

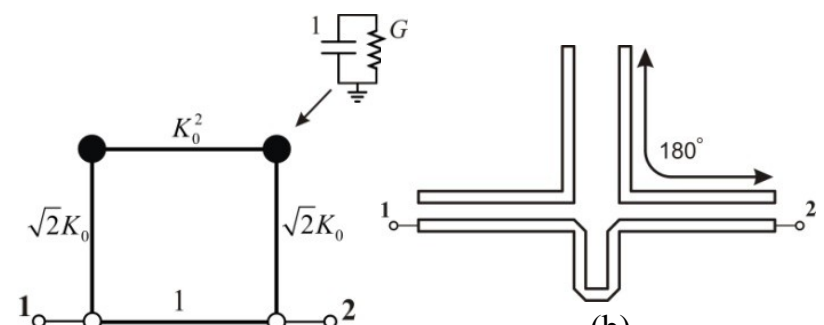

(a)

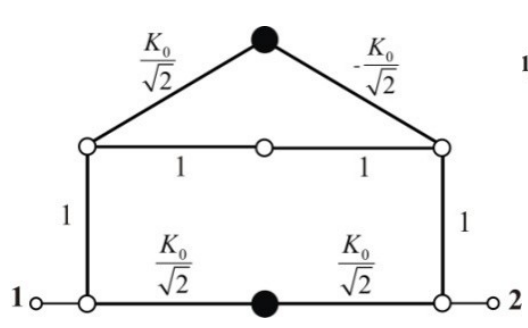

(c) (d)

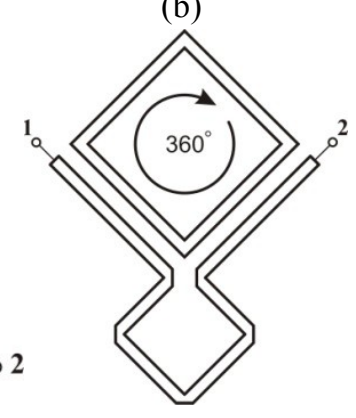

Fig. 3. (a) 1st-order allpass prototype based on Fig. 2b. (b) Microstrip realization using half-wavelength resonators. (c) 1st-order allpass prototype based on Fig. 2d. (d) Microstrip realization using a dual-mode ring resonator.

$Y_{e}$ and $Y_{o}$ form the admittance inverter network shown in Fig. 2b. Shown in Fig. 2c and Fig. 2d are networks obtained from setting $\emptyset$ equal to $\pi$ and $2 \pi$, respectively. Shown in Fig. 3 are 1st-order allpass networks ( $Y_{S}$ is a single resonator) and representative microstrip realizations. Fig. $3 b$ is the same topology used in [2]-[3] to realize absorptive notch filters.

The design of allpass networks is therefore essentially a matter of designing a one-port subnetwork of the general form shown in Fig 2a which gives the desired response, and constructing the complete allpass network according to Fig. $2 \mathrm{~b}-\mathrm{d}$. It is interesting to note that the allpass prototype networks shown in Fig. 2 are essentially admittance-scaled $90^{\circ} 3-\mathrm{dB}$ couplers. This approach also works for general values of $\emptyset$, and the resulting networks may prove to be useful as compact $(\varnothing<\pi / 2)$ allpass networks, tunable filters, or novel 3-dB couplers. Selecting values of $A<1$ is useful for dissipative loss compensation as discussed in the next section.

\section{DissiPATIVE LOSS COMPENSATION}

Filter selectivity degrades when lossy resonators are used. Selectivity can be improved by trading off insertion loss, which is usually achieved with the technique of predistortion. However, predistorted filters of any significant order $(>2)$ are asymmetric [4] and so cannot be implemented using the proposed parallel-cascade allpass approach. This approach, however, does naturally allow for novel loss compensation techniques which would be difficult to implement using conventional filter design methods. 


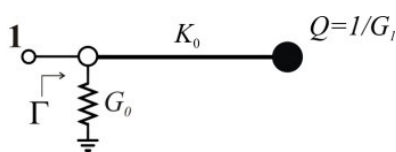

(a)

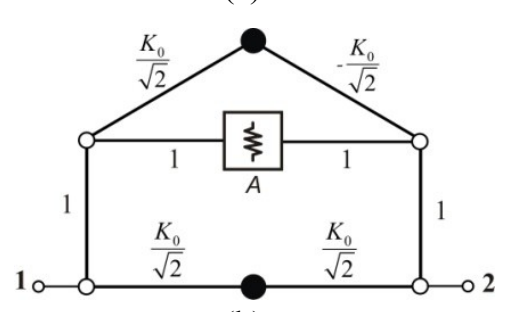

(b)

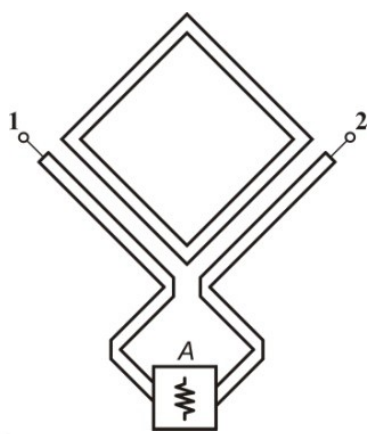

(c)
Fig. 4. (a) Subnetwork. (b) 1st-order predistorted allpass prototype. (c) Microstrip realization.

Two loss-compensation techniques applicable to the parallel-cascade allpass network approach are presented here. In the first technique, the allpass networks are individually loss compensated, and by doing so each allpass network can be made to have the same resonator $Q_{u}$. In the second technique, a certain class of lossy bandpass transfer function is used, which in general requires allpass networks with nonuniform resonator $Q_{u}$ 's.

\section{A. Predistorted Allpass Networks}

A predistorted allpass network is defined here as a network containing finite- $Q_{u}$ components, for which $\left|S_{12}\right|^{2}$ is equal to a constant value, less than unity, for all frequencies. Such networks are realizable for any order by applying the approach described in [5] to allpass transfer functions. As an example, consider a lossless first-order lowpass prototype allpass function, which can be written in the form of a reflection coefficient of a single resonator with input coupling $K$ :

$$
\Gamma=\frac{p-K^{2}}{p+K^{2}}
$$

Let $\emptyset=2 \pi$ and $A$ be an arbitrary value less than 1 and use (5) and (7):

$$
Y_{\text {in }}=\frac{1-A \Gamma}{1+A \Gamma}=G_{0}+\frac{K_{0}^{2}}{G_{1}+p}
$$

where:

$$
G_{0}=\frac{1-A}{1+A}, \quad K_{0}=K \sqrt{1-G_{0}^{2}}, \quad G_{1}=G_{0} K^{2}
$$

The resulting subnetwork described by (11) is shown in Fig. 4a. Shown in Fig $4 \mathrm{~b}$ is the complete network. Note that the subnetwork admittance $G_{0}$ has formed a matched attenuator with attenuation $A$. Shown is Fig. $4 \mathrm{c}$ is an example microstrip design. This approach is applicable to both bandpass and bandstop filters, although the attenuator adds extra loss, which becomes increasingly significant as the number of cascaded allpass networks is increased.

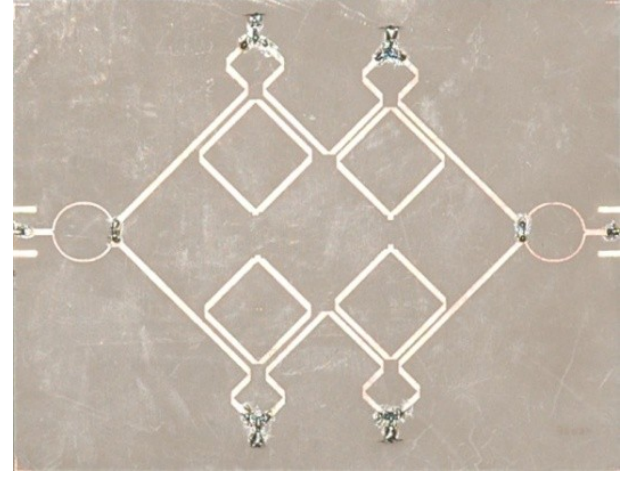

(a)

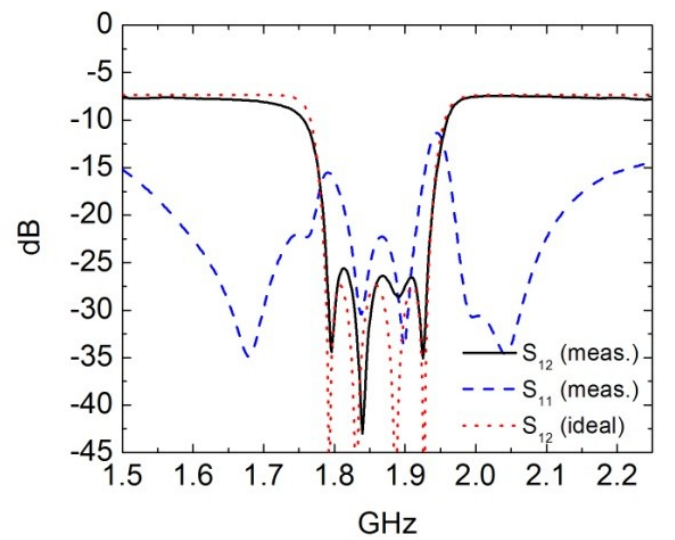

(b)

Fig. 5. (a) Bandstop microstrip prototype. (b) Measured results.

\section{A. Nonuniform $Q_{u}$ Bandpass Functions}

Highly-selective lossy symmetrical bandpass transfer functions, realizable by the parallel-cascade allpass approach, can be generated by the following procedure:

1) Begin with a lossless symmetrical bandpass transfer function.

2) Calculate $S_{e}$ and $S_{o}$, which will be in the general form of (3) and (4).

3) Shift only the zeros of $S_{e}$ and $S_{o}$ to the left in the complex plane to add loss. This is done in an adaptive fashion such that when $S_{e}$ and $S_{o}$ are recombined to form $S_{12}$, the shape of the passband is minimally affected, with a constant amount of added passband insertion loss. As the poles of $S_{e}$ and $S_{o}$ are not shifted, the poles of $S_{12}$ are unaffected by this operation. The advantage of this technique is that unlike the predistorted allpass technique just discussed, no extra loss is added.

\section{RESULTS}

Loss-compensated bandstop and bandpass filters were built and tested. The bandstop filter utilizes predistorted allpass networks, and the bandpass filter realizes a non-uniform $Q_{u}$ transfer function. The filters are microstrip fabricated on Rogers RT Duroid 6010 (thickness $1.27 \mathrm{~mm}, \varepsilon_{\mathrm{r}}=10.2$ ), and 
both filters are designed to give an ideal lossless 4th-order Chebychev response with 20-dB ripple, offset by $6 \mathrm{~dB}$.

\section{A. Bandstop Prototype}

Shown in Fig. 5a is the bandstop microstrip prototype. Wilkinson power dividers are used, and each pole/zero pair is realized with the topology of Fig 4c. $A$ is chosen for each predistorted allpass section such that the resonator $Q_{u}$ 's are equal $(\sim 200)$. Shown in Fig. $5 \mathrm{~b}$ are the measured results compared to an ideal bandstop response. The measured passband insertion loss is $7.2 \mathrm{~dB}$, the center frequency is 1.873 $\mathrm{GHz}$, and the 3-dB bandwidth is $179 \mathrm{MHz}$.

\section{B. Bandpass Prototype}

Shown in Fig. 6a is the bandpass microstrip prototype. A broadband $180^{\circ}$ coupler is used at the input in order to improve the stopband bandwidth, and a Wilkinson power divider is used at the output. The resonator $Q_{u}$ 's are approximately 200 and 80 , where the low- $Q_{u}$ resonators are implemented with patterned microstrip lines (Fig. 6b). Shown in Fig. $6 \mathrm{c}$ are the measured results, and shown in Fig. $6 \mathrm{~d}$ is the measured passband response compared to a normalized ideal response and a conventional lossy filter with the same average resonator $Q_{u}$. The measured passband insertion loss is $6.9 \mathrm{~dB}$, the center frequency is $1.9 \mathrm{GHz}$, and the $3-\mathrm{dB}$ bandwidth is 78.2 $\mathrm{MHz}$.

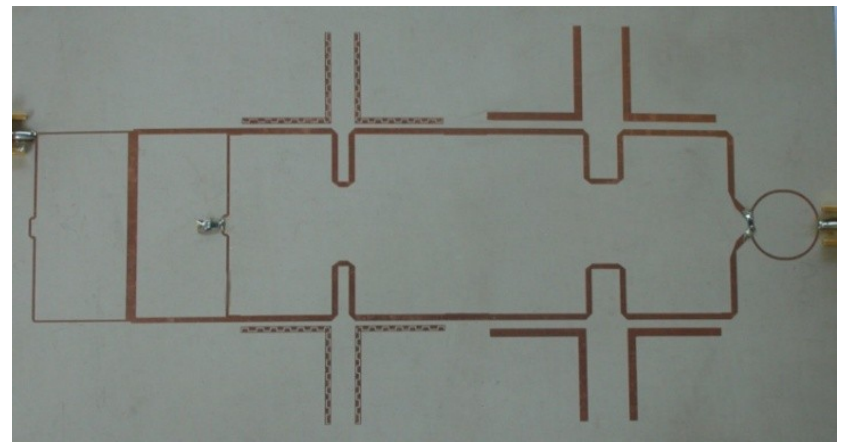

(a)

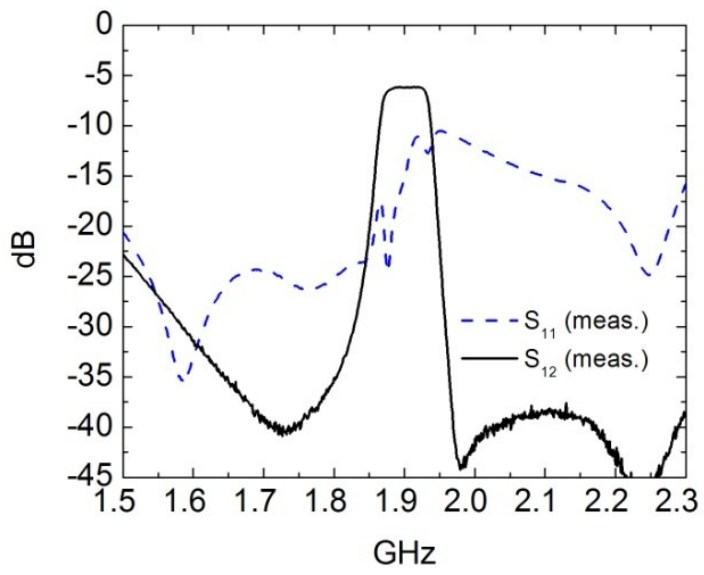

(c)

\section{CONCLUSION}

A new absorptive filter design approach using microwave allpass networks in a parallel-cascade configuration has been presented. This approach readily allows for the realization of complex filter functions, and is ideally suited for loss compensation. Two new loss compensation techniques, along with corresponding microstrip prototypes, are presented: predistorted allpass networks and nonuniform $Q_{u}$ bandpass transfer functions. Of the two techniques the latter is more useful, as no extra loss is added.

\section{REFERENCES}

[1] J. Helsajn, Synthesis of Lumped Element, Distributed, and Planar Filters. New York: McGraw-Hill, 1990.

[2] A. C. Guyette, I. C. Hunter, R. D. Pollard, and D. R. Jachowski, "Perfectly-Matched Bandstop Filters using Lossy Resonators," 2005 IEEE MTT-S Int. Microwave Symp. Dig., WE1F-2, June 2005.

[3] D. R. Jachowski, "Compact, Frequency-Agile, Absorptive Bandstop Filters," 2005 IEEE MTT-S Int. Microwave Symp. Dig., WE1F-1, June 2005.

[4] I. Hunter, Theory and Design of Microwave Filters. London: IEE, 2001.

[5] A. C. Guyette, I. C. Hunter, and R. D. Pollard, "Exact Synthesis of Microwave Filters with Nonuniform Dissipation," 2007 IEEE MTT-S Int. Microwave Symp. Dig., pp. 623-626, June 2007.

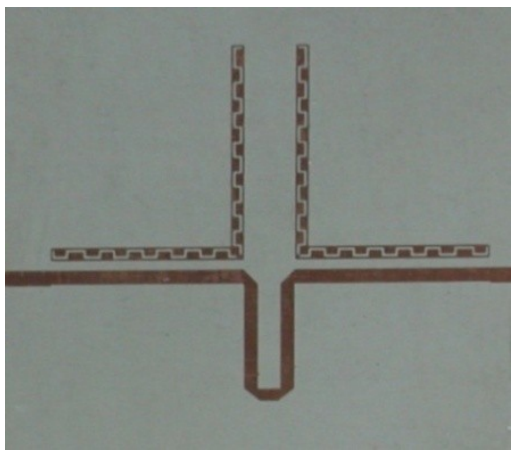

(b)

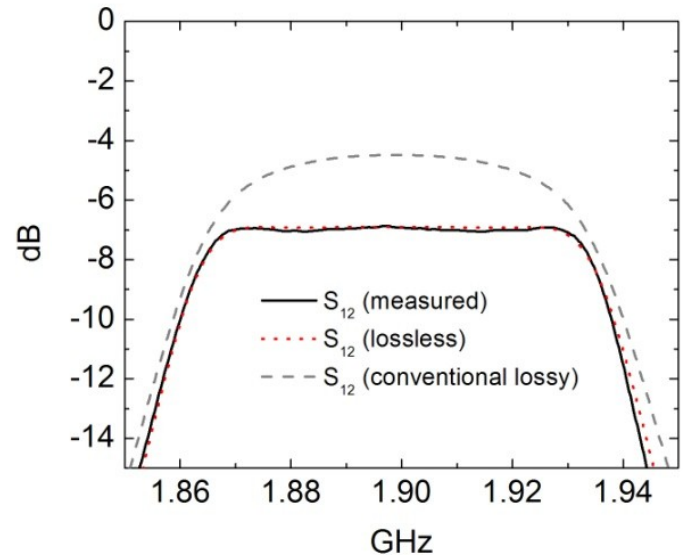

(d)

Fig. 6. (a) Bandstop microstrip prototype. (b) Detail of low- $Q_{u}$ allpass network. (c) Measured results. (d) Comparison of measured passband response, ideal normalized response, and conventional lossy response. 